\title{
The Bacteriomes of Ileal Mucosa and Cecal Content of Broiler Chickens and Turkeys as Revealed by Metagenomic Analysis
}

\author{
Shan Wei, Michael Lilburn, and Zhongtang Yu \\ Department of Animal Sciences, The Ohio State University, Columbus, OH 43210, USA \\ Correspondence should be addressed to Zhongtang Yu; yu.226@osu.edu
}

Received 9 August 2016; Revised 7 November 2016; Accepted 27 November 2016

Academic Editor: Akira Hiraishi

Copyright (C) 2016 Shan Wei et al. This is an open access article distributed under the Creative Commons Attribution License, which permits unrestricted use, distribution, and reproduction in any medium, provided the original work is properly cited.

\begin{abstract}
The gastrointestinal (GI) bacteriome of poultry is important in host nutrition and health, but its diversity and composition remain poorly characterized. In this study we phylogenetically characterized the bacteriome in the cecal contents and ileal mucosa of chickens and turkeys using metagenomics empowered by pyrosequencing technique. $>95 \%$ coverage of bacterial diversity was achieved except for the turkey ileal mucosa. Collectively, 3,401 and 125 operational taxonomy units (OTU, defined at a 0.03 phylogenetic distance) in chicken, and 1,687 and 16 OTUs in turkey were identified from the cecal content and the ileal mucosa, respectively. Besides those previously reported, 39 and 50 additional genera of bacteria were identified in the chicken and turkey cecal bacteriome, respectively. Although the GI bacteriomes of the same region in both species exhibited greater similarity than the bacteriomes of different regions within each species, broiler chickens and turkeys harbor a distinct intestinal bacteriome. Such difference may suggest different dietary interventions for bacteriome modulation for enhanced nutrient utilization and gut health. The results may also be useful in developing prebiotics, probiotics, and analytical tools (e.g., phylochips). We also determined the variation in the number of OTUs and variability between two independent pyrosequencing runs and two data processing pipelines.
\end{abstract}

\section{Introduction}

The poultry gastrointestinal (GI) tract harbors a dynamic microbial community consisting of a large number of species, primarily bacteria [1]. This bacterial community, or bacteriome, plays a pivotal role in the overall health and performance of poultry. The GI bacteria can be roughly classified as either pathogenic or commensal organisms [2]. Pathogenic bacteria can harm the host by causing localized or systemic infections and intestinal lesions [3] while commensal bacteria can benefit the host by providing nutrients, metabolic facilitation, and competitive exclusion $[4,5]$. A better understanding of the bacterial composition and activity as well as the underlying mechanisms by which indigenous bacteria modulate the GI environment is needed to improve host health and feed utilization.

For many years, studies aimed at understanding the poultry GI bacteriome relied on classical cultivation techniques. During the past two decades, however, the $16 \mathrm{~S}$ rRNA gene has been used as a primary biomarker for bacterial identification in various environments including the GI tract of poultry. This technique overcomes the limitation of culture-dependent methodologies thus potentially allowing for the identification of all the GI bacteria irrespective of their culturability. Studies using individual $16 \mathrm{~S}$ rRNA gene clone libraries have provided valuable insight into the diverse GI bacteriome of poultry by producing highquality sequences. However, these studies were limited to relatively small numbers of sequences that were affordable to researchers. Consequently, a comprehensive study looking into the diversity and composition of the GI bacteriome in poultry was not possible until next-generation sequencing (NGS) becomes available.

High-throughput NGS technologies have proven to be powerful tools for comprehensive analysis of complex bacteriomes $[6,7]$. NGS technologies can generate large amounts of sequencing data at a relatively low cost. It also allows for sequencing of environmental DNA without a prior cloning step thereby eliminating cloning bias $[8,9]$. The unprecedented sequencing capacity also allows for the identification of bacteria that are present in low abundance in a bacteriome. Although being replaced by other NGS technologies, the 
TABLE 1: Barcoded degenerate primers used to produce the V3 amplicon libraries.

Forward

Reverse

The unique barcode is underlined.

454 pyrosequencing technology is the first NGS technology that has been widely used to analyze the GI bacteriomes of human and animals as well as environmental bacteriomes [10-12]. However, to date, there are only a few studies that have attempted to characterize the diversity and composition of the GI bacteriome of chickens and turkeys [13-16]. These studies only reported a relatively small number of sequences per sample $(<10,000$ reads) resulting in low coverage and a still incomplete picture of the diversity within the poultry GI bacteriome.

It has been recognized that the 454 pyrosequencing technology generates sequencing errors, sequence artifacts, and chimeric sequences $[18,19]$. Also, different 454 sequence analysis pipelines use different algorithms for sequence alignment, phylogenetic distance computation, and OTU clustering, leading to different results [18-22], including over- or underestimation of diversity and species (OTUs) richness [23]. Moreover, little attention has been given to the repeatability of the technology across different pyrosequencing runs even though variability between runs can occur [24]. Thus, the primary objective of this study was to characterize the composition of the GI bacteriome in commercial poultry species, (chicken and turkey) using the 454 pyrosequencing technique. The secondary objective was to evaluate the repeatability of the technique and the effects of different data processing pipelines.

\section{Materials and Methods}

2.1. Sample Collection. All animal protocols were approved by the Ohio Agricultural Research and Development Center Animal Care and Use Committee.

Five broiler chickens were randomly chosen from each of three flocks at six weeks of age, and eight turkeys were chosen from one flock at 14 weeks of age. Both the chicken flocks and the turkey flock reared at the poultry Research Farm located at the Ohio Agricultural Research and Development Center (OARDC), Wooster, Ohio. The chickens and turkeys were fed standard corn-soybean-meal-based diets that met or exceeded the NRC requirements [25] for each species. Cecal contents were collected from each bird and pooled by species (the samples were pooled to reduce the number of samples while achieving high depth coverage per sample). The sequencing was done before Illumina sequencing was available. Individual ileal mucosa samples were collected from the region between Meckel's diverticulum and the ileocecal junction and pooled within species as described previously [26]. Each composite sample was mixed to represent each species and each GI region.
2.2. DNA Extraction and PCR. Community DNA was extracted from each of the four composite samples using the repeated bead-beating plus column purification method [27]. The V3 region (about $200 \mathrm{bp}$ in length) of $16 \mathrm{~S}$ rRNA gene in the metagenomic DNA was amplified with barcoded universal primer sets as listed in Table 1. Each forward primer consists of three parts: a 19 nt degenerated universal primer for bacterial 16S rRNA gene (357F), a $10 \mathrm{nt}$ barcode, and the pyrosequencing adapter A. The reverse primer consisted of an $18 \mathrm{nt}$ degenerated universal primer for bacterial $16 \mathrm{~S}$ rRNA gene (519R) and a $19 \mathrm{nt}$ pyrosequencing adapter $\mathrm{B}$. The degenerate bases on primers were introduced to expand their inclusiveness.

For each PCR reaction, $400 \mathrm{ng}$ of metagenomic DNA template was added to a $49 \mu \mathrm{L}$ master mix that contained 1x PCR buffer, $1.75 \mathrm{mM} \mathrm{MgCl}_{2}, 670 \mathrm{ng} / \mu \mathrm{L}$ bovine serum albumin, $200 \mu \mathrm{M}$ dNTP, $500 \mathrm{nM}$ of each primer, and $0.625 \mathrm{U}$ Platinum Taq DNA polymerase (Invitrogen Corporation, Carlsbad, CA). The PCR thermal program consisted of an initial denaturation at $95^{\circ} \mathrm{C}$ for $10 \mathrm{~min} ; 20$ or 25 cycles $(20$ cycles for cecal content samples and 25 cycles for ileal mucosa samples) of a $30 \mathrm{~s}$ denaturation step at $95^{\circ} \mathrm{C}$, a $35 \mathrm{~s}$ annealing step at $55^{\circ} \mathrm{C}$, and a $35 \mathrm{~s}$ elongation step at $72^{\circ} \mathrm{C}$; and a final extension step at $72^{\circ} \mathrm{C}$ for $7 \mathrm{~min}$, before a $4^{\circ} \mathrm{C}$ hold.

The quality of the PCR products was examined using agarose (1.2\%) gel electrophoresis, and the expected PCR products of approximately $200 \mathrm{bp}$ were gel purified using a Qiagen Gel Purification Kit (Qiagen, Valencia, CA, USA). The concentration of the purified products was quantified using a NanoDrop ND-1000 spectrophotometer (Thermo Scientific, Wilmington, DE) and confirmed using a Quant-it Kit (Invitrogen Corporation, Carlsbad, CA, USA).

2.3. Pyrosequencing and Data Analysis. Given the expected higher diversity in the cecal content samples compared with the ileal mucosa samples, the amplicons from the former and the latter were mixed in a 9:1 ratio for each species. The amplicons from the chickens and the turkeys were subsequently pooled in a 2:1 ratio. The pooled amplicon samples were divided and sequenced in two independent pyrosequencing runs on one-half of a picotiter plate each using a 454 Life Sciences Genome Sequencer FLX system (Roche, Basel, Switzerland) before the Genome Sequencer FLX Titanium became available. The V3 hypervariable region was sequenced because the FLX system produces a read length of less than $250 \mathrm{bp}$. The raw data were provided as sff files.

The quality of the 454 pyrosequencing data was evaluated using the raw sff data files following the standardized 
operating procedure (SOP) proposed by Schloss et al. [19]. Briefly, the flow file was generated from the sff file of each sample using the sffinfo program of the GS Analysis Software (Version 2.5, 454 Life Sciences Corporation, Branford, CT, USA). The pyrosequencing noise of each flow file was removed by the AmpliconNoise function implemented in Mothur $[18,19,28]$. The "denoised" sequences were trimmed off the primer $357 \mathrm{~F}$ and $519 \mathrm{R}$, which results in sequences of the V3 hypervariable region of $16 \mathrm{~S}$ rRNA genes (minimum length: $100 \mathrm{bp}$, average length: $145 \mathrm{bp}$ ). The trimmed sequences were aligned using the Mothur aligner [28, 29] with the Silva_SSU_Ref_NR_108 dataset [30] as reference sequences and with a -4 score penalty for gap-pen and -3 score penalty for mismatch. Sequences that could not be aligned with the Silva reference dataset were removed. The common gaps in the sequence alignment were filtered out, and the sequences were preclustered to remove sequences that contain possible pyrosequencing errors [19, 31]. Possible chimeric sequences were identified and removed using UCHIME [32] implemented in the Mothur package [28].

A distance matrix of each dataset was computed using the ARB database environment with the Jukes-Cantor correction [30] applied. The Mothur and USEARCH were used to cluster sequences into OTUs at $0.03,0.05$, and 0.20 phylogenetic distances, generate rarefaction curves, and determine the nonparametric ACE and Chaol estimates of maximum richness from each of the distance matrices. The maximum number of OTUs likely present in each of the samples was also estimated using the nonlinear models procedure (PROC NLIN) of SAS (V9.2, SAS Inst. Inc., Cary, NC), which fits the monomolecular function to the rarefaction output to determine the asymptote that serves as the upper bound of the curves as previously described [33]. Each distance matrix was computed three times, and the median values were chosen in calculating these indices to avoid under- or overestimation.

One representative sequence of each OTU defined at 0.03 genetic distances was obtained using the "get.oturep" command in the Mothur package V1.22. [28]. These OTU representative sequences were imported into $\mathrm{ARB}$, and then a phylogenetic tree was constructed for each sample by inserting each sequence into the reference tree of the 286,858 Silva reference sequences (SSU_111_Ref_NR, http://www.arb-silva.de) as described previously [34]. The phylogenetic tree was then used for weighted UniFrac analysis as described in library comparison below. The sequences used in this study are maintained in an in-house ARB database dedicated to the GI bacteriome of chickens and turkey and is available from the corresponding author. The representative sequences of each sample were also archived in the MG-RAST server under the project of Poultry_MID_DB (4508915.3 to 4508920.3). The OTUs were classified using the RDP classifier [35] and the composition of each bacteriome was visualized as a taxonomic tree constructed using MEGAN [36]. Briefly, the taxonomic identification of $16 \mathrm{~S}$ rRNA sequences was performed using the RDP naive Bayesian classifier on the RDP server (https://rdp.cme.msu.edu/) [35] with the default setting. The taxonomy files were retrieved and imported into MEGAN using a minimum cutoff of 5 OTUs and a confidence score $\geq 50.0 \%$ [36]. The bacteriome composition of each gut location was shown as a hierarchical tree with the nodes showing the OTU counts (Figures 1-4). The bacteriome composition of cecal content was also compared between the chickens and the turkeys (Figure 5).

The GI bacteriomes of chickens and turkeys were compared using 4 different methods: weighted UniFrac distance, which measures the phylogenetic distance between sets of taxa as phylogenetic trees [37, 38]; the SONS function [39] in the Mothur package [28], which compares two bacteriomes by taking into consideration OTU richness, membership, and structure; RDP library comparison [40], which compares two libraries side by side based on the represented taxa and computes the likelihood that the frequency of membership in a given taxon is the same; and MEGAN phylogenetic tree [36], which allows comparison of bacteriomes based on detailed phylogenetic composition.

The numbers of raw sequence reads and quality-checked sequences were compared between the two independent pyrosequencing runs to assess the variation between the two runs. The quality-checked sequences were aligned using two different aligners in parallel to assess the effect of different aligners on OTUs clustering: the RDP Pyro aligner (http://pyro.cme.msu.edu/) against the Silva reference dataset provided on Mothur's webpage (https://www.mothur.org/) and the Mothur aligner. One distance matrix was computed for each alignment with the Jukes-Cantor correction applied, and OTUs were clustered using Mothur.

\section{Results and Discussion}

3.1. Overview of the 454 Pyrosequencing Results and the Variation between Runs. A total of 402,247 DNA sequence reads were obtained, of which 338,177 were successfully assigned to the corresponding samples based on the barcodes (Table 2). The sequencing data from the two pyrosequencing runs did not differ significantly regarding the number of raw sequences or sequences resulting from the denoising step. However, after the preclustering step, which was designed to reduce the effect of pyrosequencing errors [31], the second pyrosequencing run resulted in approximately 55\% fewer sequence reads and $82 \%$ fewer denoised sequences. The coverage of diversity and composition of the GI bacteria also differed between the two pyrosequencing runs on the same sample set even though the numbers of raw reads were very similar. These results suggest that considerable variability in sequencing quality and numbers of usable sequences between different runs of the same pyrosequencing system corroborate a previous report [24]. Because the same amplicon libraries were used, the variability was produced during the sequencing process. Such run-to-run variations were also reported for the MiSeq technology [41].

The RDP Pyro aligner and Mothur aligner both align 16S rRNA sequences based on their secondary structure and can align a massive number of sequences relatively quickly [23, 29, 40, 42]. The RDP Pyro aligner does not align the hypervariable regions while the Mothur aligner does using the Silva alignment as reference sequences. Both aligners have been commonly used in analysis of pyrosequencing data. 


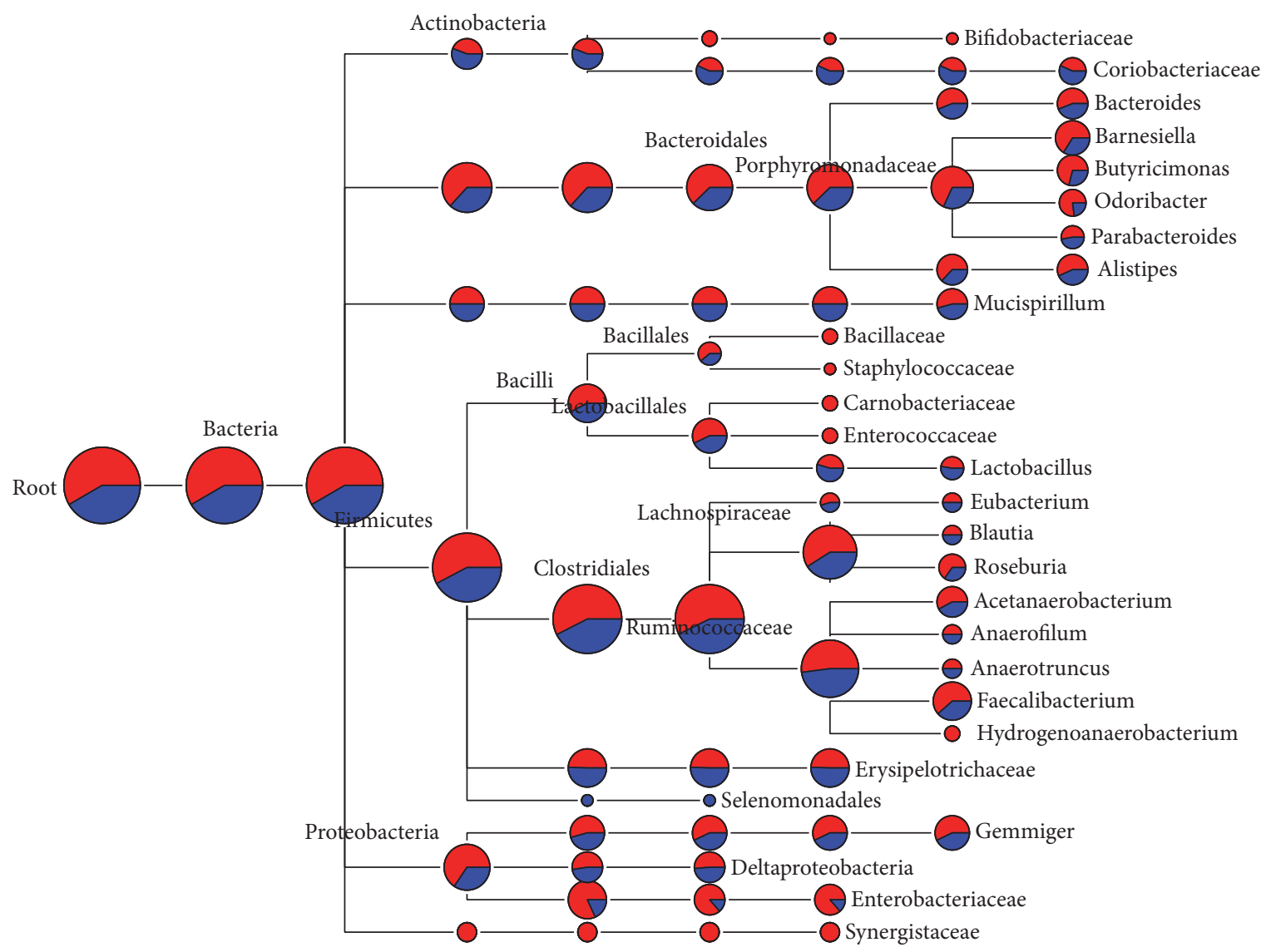

FIGURE 1: Bacterial diversity of chicken cecal content bacteriome. Only the genera represented by $\geq 5$ OTUs each were shown, and the size of each node reflects the total number of OTUs. The relative proportion of taxa from the two pyrosequencing runs was shown by different colors: red, from the first run; blue, from the second run.

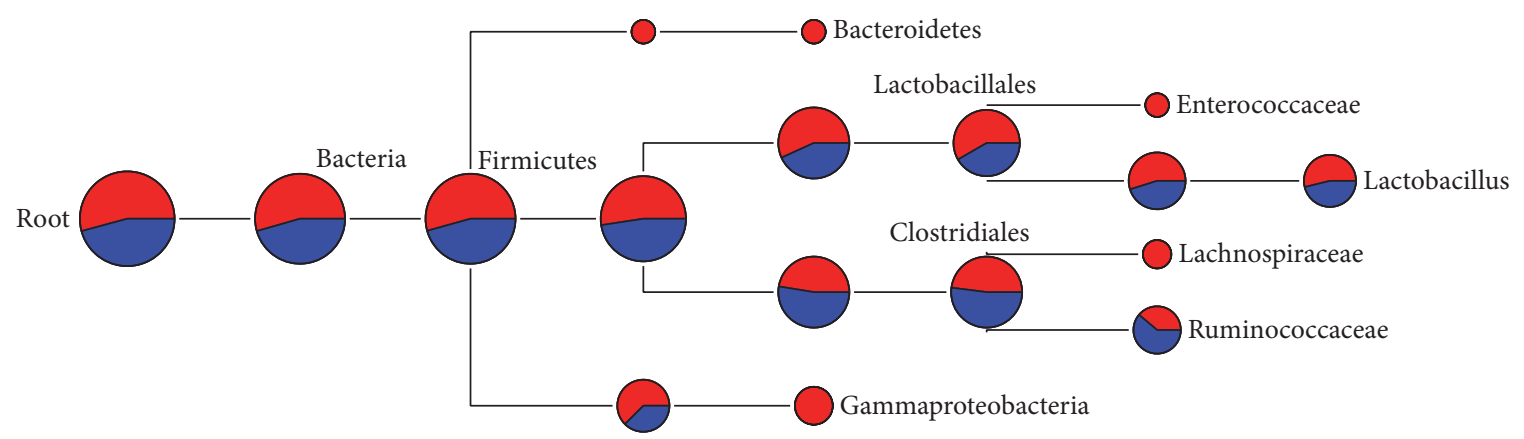

FIGURE 2: Bacterial diversity of chicken ileal mucosal bacteriome. Only the genera represented by $\geq 5$ OTUs each were shown, and the size of each node reflects the total number of OTUs. The relative proportion of taxa from the two pyrosequencing runs was shown by different colors: red, from the first run; blue, from the second run.

Thus, the two aligners were evaluated using the same dataset. The Mothur aligner resulted in approximately twice as many OTUs with the default alignment setting when penalties were given to gap open and mismatch. The RDP aligner yielded > 3-fold more OTUs than the Mothur aligner when the penalty options were applied. The RDP aligner has been reported to result in more OTUs from all but the V3 and V4 hypervariable regions of $16 \mathrm{~S}$ rRNA genes when compared to the Silva aligner [19]. In this study, the V3 region was used, and more OTUs also resulted from the RDP Pyro aligner. In addition, even though the SOP of Mothur includes several quality checking procedures, the default setting may not be optimal for every dataset. As demonstrated in this study, the introduction of penalties for gap open and mismatch can significantly reduce the likelihood of overestimating diversity. It is recommended that in future studies more than one setting be used in each step to avoid inflation of diversity. Hence, the Mothur aligned sequences with penalties for gap open and mismatch 


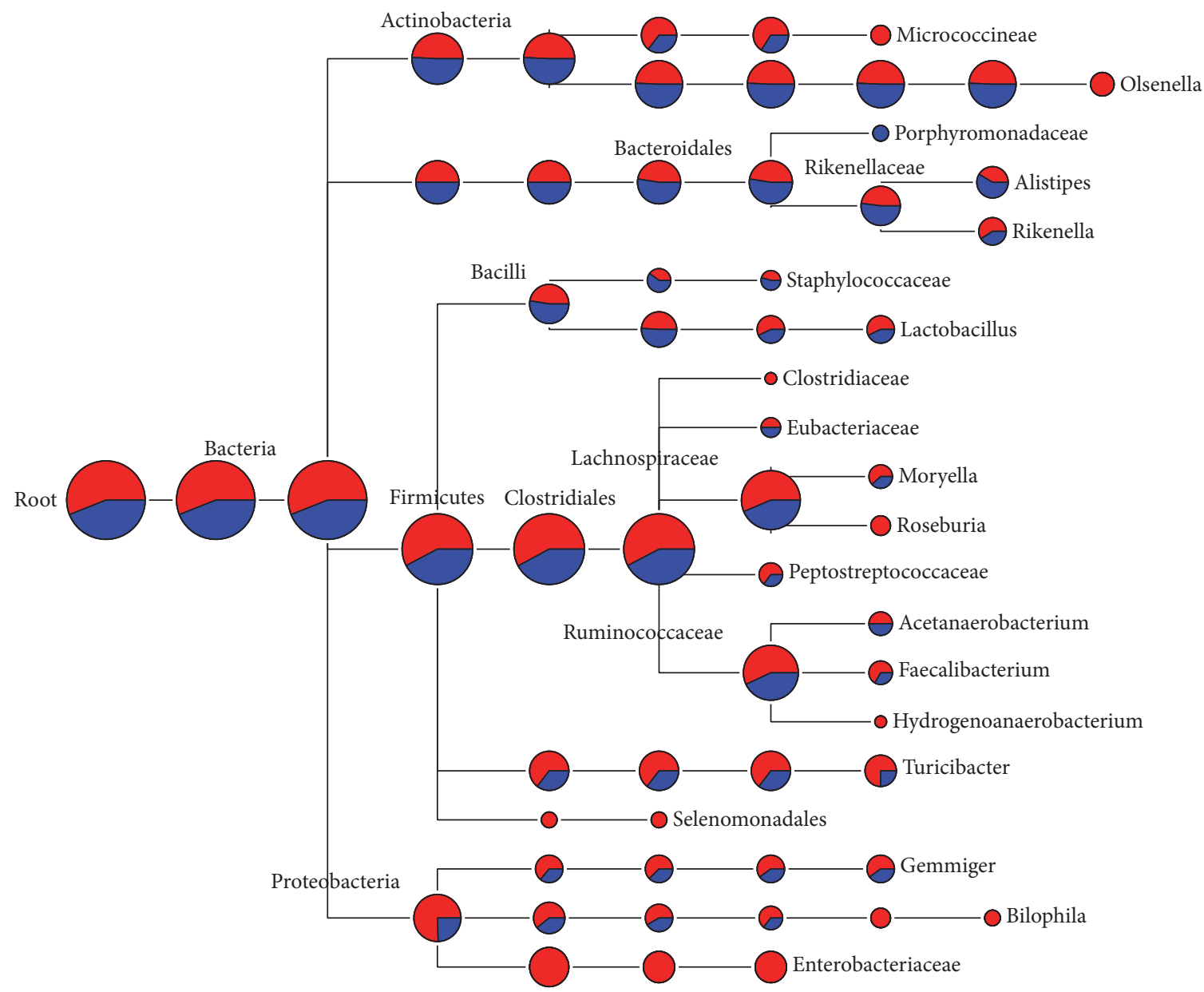

FIGURE 3: Bacterial diversity of turkey cecal content bacteriome. Only the genera represented by $\geq 5$ OTUs each were shown, and the size of each node reflects the total number of OTUs. The relative proportion of taxa from the two pyrosequencing runs was shown by different colors: red, from the first run; blue, from the second run.

applied were used in assessing the effect of different clustering algorithms on OTUs clustering in the present study.

Species richness of metagenomic datasets is typically expressed as numbers of OTUs clustered at a specific phylogenetic distance (commonly 0.03). In this study, Mothur and USEARCH, both of which are commonly used in OTU clustering $[28,43,44]$, were compared using the same dataset of all four bacteriome samples. The USEARCH method generated twice as many OTUs as Mothur (Table 2). These results suggest that different clustering methods can produce different estimates of species richness, and thus comparisons of results from various studies, especially those that used different clustering and alignment methods, should be done with caution. Additionally, different phylogenetic distances might be needed when different clustering methods are used to produce comparable species richness. In this study, the Mothur was used to cluster OTUs from all the four datasets because it probably did not overestimate species richness.

\subsection{Bacterial Diversity of Chicken Cecal Content Bacteriome.} The cecum is the largest major reservoir of bacteria in poultry. The first and the second pyrosequencing runs produced 3,973 and 2,829 OTUs, respectively, from the chicken cecal samples
(Table 2). The estimated asymptotes of OTUs reached 1.5-fold of the number of observed OTUs. Both pyrosequencing runs achieved a high level of coverage (Good's coverage $>95 \%$ ) of the bacterial diversity in the chicken cecal bacteriome. The RDP classification of the OTUs from both pyrosequencing runs was combined and imported into MEGAN to generate a taxonomic tree of the major bacteria (Figure 1). In total, we identified nine bacterial phyla (Firmicutes, Proteobacteria, Bacteroides, Synergistetes, Fusobacteria, Actinobacteria, Deferribacteres, Tenericutes, and Lentisphaerae) and 84 known genera (data not shown). The Firmicutes was the most predominant phylum, accounting for $57.8 \%$ of the total bacterial sequences of the cecal content sample. Within this phylum, $30.9 \%$ of the OTUs could not be classified to any known taxa. The Bacteroidetes and Proteobacteria were far less predominant, accounting for $5.4 \%$ and $4.3 \%$ of the total bacteria sequences, respectively. No significant difference was observed at phylum or class level between the two pyrosequencing runs except for the class Gammaproteobacteria. However, a major difference was observed in unclassified Enterobacteriaceae. In the first pyrosequencing run, Bifidobacteriaceae, Bacillaceae, Staphylococcaceae, Carnobacteriaceae, Enterococcaceae, Hydrogenoanaerobacterium, and 


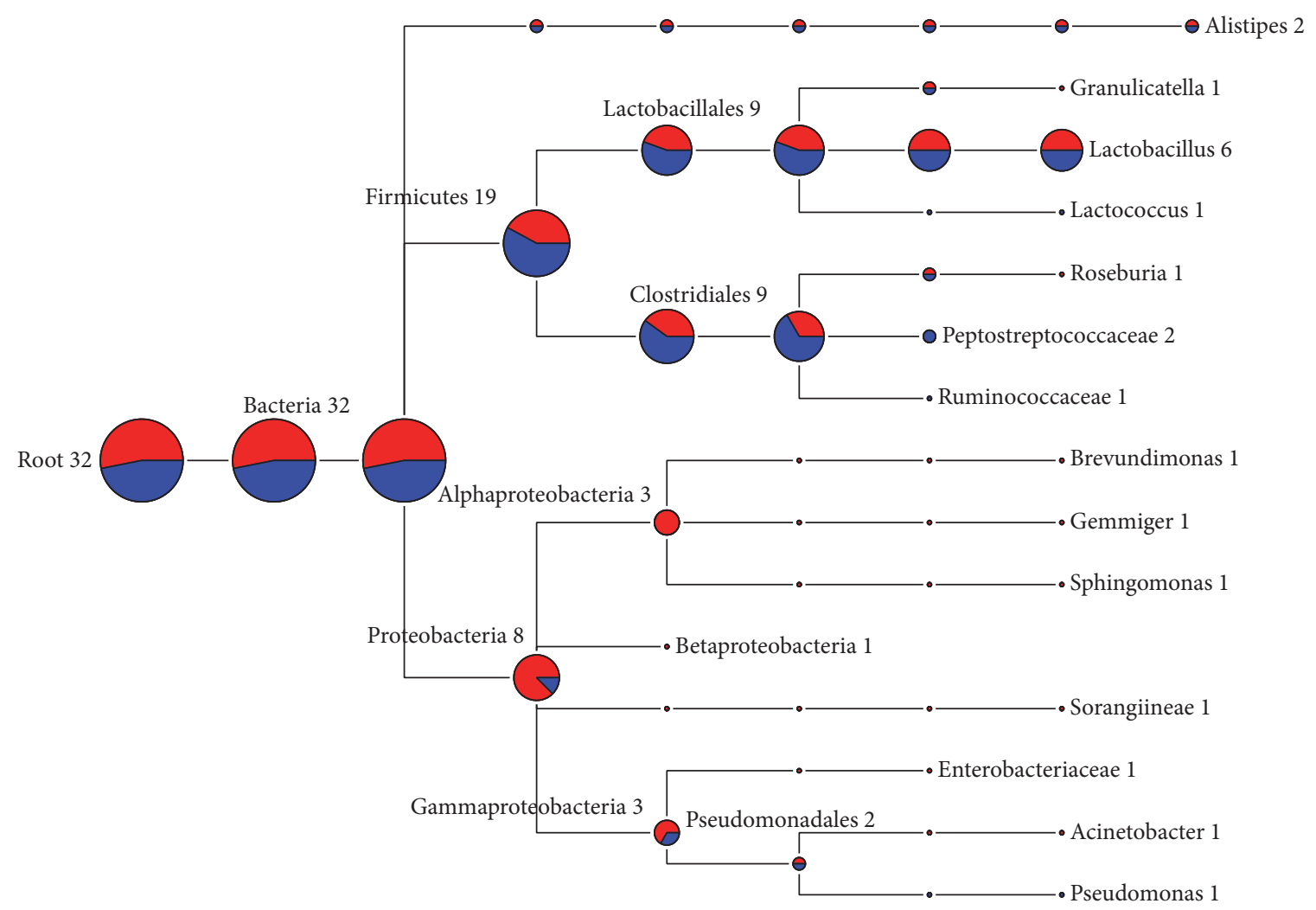

Figure 4: Bacterial diversity of turkey ileal mucosal bacteriome. Only the genera represented by $\geq 5$ OTUs each were shown, and the size of each node reflects the total number of OTUs. The relative proportion of taxa from the two pyrosequencing runs was shown by different colors: red, from the first run; blue, from the second run.

Synergistaceae were found to be represented by $\geq 5$ OTUs each, while Selenomonadales was the only taxon that was represented by $>5$ OTUs. Consistent with a previous study [24], noticeable variation in diversity estimates can arise from different runs and quantitative interpretation of pyrosequencing data should be done with caution. Because the same amplicon libraries were sequenced in the two pyrosequencing runs and the sequence data were analyzed using the same sequence pipeline and parameters, the variations between the two pyrosequencing runs probably have arisen from the sequencing process.

A recent study established a global diversity framework of the poultry GI bacteriome by using a naïve analysis of all the $16 \mathrm{~S}$ rRNA gene sequences generated from poultry GI (primarily cecal) bacteria that have been recovered worldwide using the Sanger sequencing technology [34]. When compared to this global cecal bacterial diversity database, there were 45 genera of bacteria in that database that were also found in the present study (Supplementary Table 1, in Supplementary Material available online at http://dx.doi.org/10.1155/ 2016/4320412). However, 29 genera were missing from the current pyrosequencing study (Supplementary Table 2). These include Salmonella, Megamonas, and Paraprevotella, each of which was represented by $>10$ sequences in that databases. On the other hand, the current pyrosequencing study identified 39 bacterial genera that were not represented in the global cecal bacterial diversity database, including Butyricimonas, Odoribacter, Hydrogenoanaerobacterium,
Moryella, Parasporobacterium, and Ruminococcus (Supplementary Table 3). There were also many minor genera represented by less than five OTUs each. Overall, this study expanded the number of bacterial genera identified in the cecum of chickens by $80 \%$. This is likely attributed to the increased sequencing depth we intentionally achieved. Most of these new genera found in the cecum might be present at low abundance. Future studies can further elucidate their importance to the host.

\subsection{Bacterial Diversity of Chicken Ileal Mucosal Bacteriome.} In this study, $>5,000$ sequences were obtained from each of the two pyrosequencing runs, resulting in 135 and 114 OTUs in the first and second runs, respectively (Table 2). Although the Good's coverage also reached $>95 \%$, the estimated asymptotes of OTUs were 1.9-fold greater than the observed numbers of OTUs. These results suggest that the diversity in the ileal mucosa has not been completely identified. It should be noted that approximately half of the original sequencing reads from the ileal mucosa appeared to be $18 \mathrm{~S}$ rRNA genes of the host. The presence of these host sequences was probably due to the broad specificity of primers $357 \mathrm{f}$ and 519 r, both of which can anneal to 18S rRNA genes [45]. Bacterial domainspecific primers can reduce or eliminate amplification of host $18 \mathrm{~S}$ rRNA genes.

The OTUs from the chicken ileal mucosa were classified into seven bacterial phyla: Actinobacteria, Bacteroidetes, 


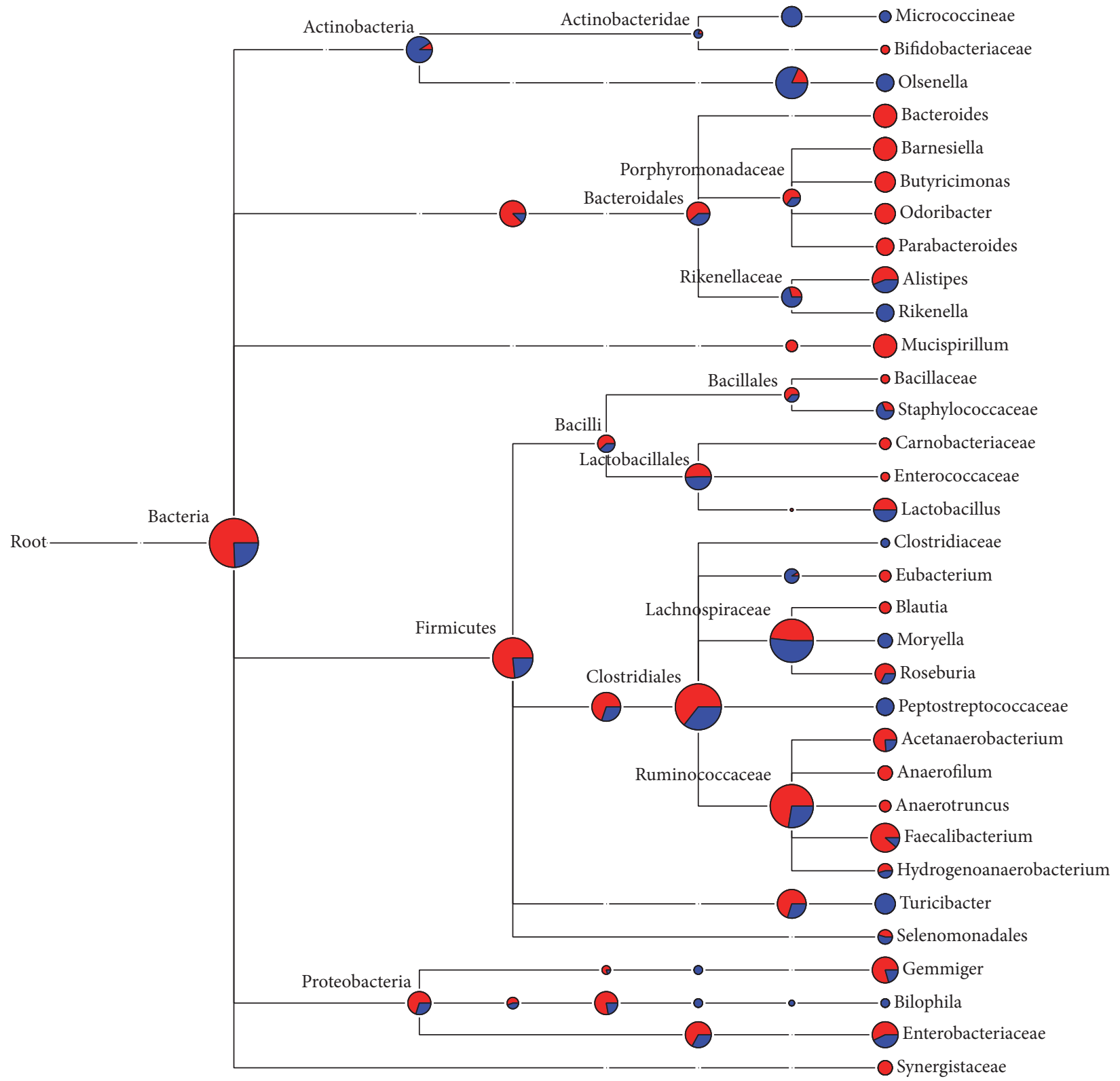

FIGURE 5: Comparison of the diversity of cecal content bacteriomes between broiler chickens and turkey. Only the genera represented by $\geq 5$ OTUs each were shown, and the size of each node reflects the total number of OTUs from the four microbiomes. The proportion of each microbiome is distinguished by color: red, chicken; blue, turkey.

Cyanobacteria/Chloroplast, Firmicutes, Proteobacteria, Synergistetes, and Saccharibacteria. Firmicutes and Proteobacteria were the major phyla, accounting for $72.6 \%$ and $11.1 \%$ of the total sequences of the chicken ileal mucosa, respectively (Figure 2). For the predominant taxa, significant differences between the two pyrosequencing runs were not observed at taxonomic ranks from phylum to genus. However, some minor groups were only identified in one of the two pyrosequencing runs. For example, Bacteroidetes, Enterococcaceae, Lachnospiraceae, and Gammaproteobacteria were only identified in the first run, and each was represented by at least 5 OTUs, whereas Prevotella, Salinicoccus, and another 10 genera, each of which was represented by one or two OTUs, were only identified in the second run. Lactobacillus was the largest genus in the chicken ileal mucosa, accounting for $11 \%$ of the total sequences. In a previous study using 16S rRNA gene clone libraries, Lactobacillus was found to account for $75 \%$ of the ileal mucosal bacterial sequences among 7-day-old chicks [26]. The differences in bird age and methodologies used might explain the discrepancy in Lactobacillus predominance witnessed in the ileal mucosa. In a study in Australia, approximately 99\% of the bacterial sequences from jejunal mucosa were classified to Lactobacillus [46]. Choices of methodology and target regions of the $16 \mathrm{~S}$ rRNA gene may influence the observed relative abundance of lactobacilli in the gut of chickens. A greater predominance of Lactobacillus was expected for the jejunum than for the ileum. 
TABLE 2: Summary of the 454 pyrosequencing data.

\begin{tabular}{|c|c|c|c|c|c|c|c|c|c|}
\hline \multirow[b]{2}{*}{ Sample } & \multirow{2}{*}{$\begin{array}{l}\text { \# of raw seqs } \\
\text { assigned }\end{array}$} & \multirow{2}{*}{$\begin{array}{l}\text { \# of sequences } \\
\text { after screening }\end{array}$} & \multirow{2}{*}{$\begin{array}{c}\text { \# of preclustered } \\
\text { seqs after } \\
\text { screening }\end{array}$} & \multicolumn{2}{|c|}{ Observed OTUs } & \multicolumn{3}{|c|}{ Maximum \# of OTUs } & \multirow{2}{*}{$\begin{array}{c}\text { Good's } \\
\text { coverage }^{* *}\end{array}$} \\
\hline & & & & Mothur & USEARCH & $\begin{array}{l}\text { Rarefaction } \\
\text { asymptote }^{*}\end{array}$ & Chaol & ACE & \\
\hline CD-1 & 98021 & 91456 & 21080 & 3973 & 8899 & 6001 & 10657 & 19318 & $95.7 \%$ \\
\hline CD-2 & 98238 & 91227 & 12437 & 2829 & 5162 & 4278 & 7806 & 14361 & $96.9 \%$ \\
\hline CM-1 & 5457 & 2714 & 598 & 135 & 324 & 259 & 370 & 1121 & $95.0 \%$ \\
\hline $\mathrm{CM}-2$ & 5273 & 2506 & 330 & 114 & 199 & 205 & 287 & 580 & $95.5 \%$ \\
\hline TD-1 & 56959 & 53527 & 10304 & 1891 & 4252 & 2779 & 5114 & 9286 & $96.5 \%$ \\
\hline TD-2 & 57442 & 49836 & 6685 & 1484 & 2706 & 2188 & 3971 & 6948 & $97.0 \%$ \\
\hline TM-1 & 8104 & 30 & 23 & 17 & 19 & 63 & 63 & 246 & $43.3 \%$ \\
\hline TM-2 & 8683 & 33 & 19 & 15 & 16 & 19 & 18 & 21 & $54.5 \%$ \\
\hline
\end{tabular}

$\mathrm{CD}$, chicken cecal digesta; CM, chicken ileal mucosa; TD, turkey cecal digesta; TM, turkey ileal mucosa.

${ }^{*}$ Estimated by Number of phylotypes $=\alpha\left(1-\beta \times e^{[-\kappa \times n]}\right)$.

${ }^{* *}$ Estimated by Coverage of diversity $=(n-N) / n \times 100 \%$.

\subsection{Bacterial Diversity of Turkey Cecal Content Bacteriome.} From the first and the second pyrosequencing runs, 1,891 and 1,481 OTUs were obtained, respectively (Table 2 ). Similar to the chicken cecal bacteriome, the estimated OTU asymptote of the turkey cecal bacteriome was approximately 1.5-fold greater than the number of OTUs observed, while the Good's coverage reached $>95 \%$. The OTUs were classified into eight bacterial phyla (Firmicutes, Bacteroides, Actinobacteria, Proteobacteria, Verrucomicrobia, Synergistetes, Elusimicrobia, and Lentisphaerae) and 85 known genera (data not shown). The phyla represented by $\geq 5$ OTUs each are shown on the taxonomy tree (Figure 3). Firmicutes, Proteobacteria, and Actinobacteria were the most predominant phyla in the turkey cecal bacteriome, accounting for $66.3 \%, 7.4 \%$, and $3.2 \%$ of the total bacterial sequences identified. Based on RDP Library comparison, a significant difference in the proportions of the phyla Firmicutes and Proteobacteria was observed between the two pyrosequencing runs. In total, 17 genera or groups were recovered only in the first sequencing run, including Olsenella, Clostridiaceae, Roseburia, Hydrogenoanaerobacterium, Selenomonadales, Bilophila, Enterobacteriaceae, and other groups, each of which was represented by $\leq 5$ OTUs (Figure 3 ). On the other hand, 14 genera or groups were identified only in the second sequencing run, including Porphyromonadaceae and other minor genera. Only one OTU was classified as Escherichia/Shigella, a common genus of enteric bacteria, and this might be due to its low abundance in the turkey cecal bacteriome. Quantitative PCR analysis for this OTU may help confirm its abundance in the turkey cecal bacteriome.

The bacterial profile of the turkey cecal bacteriome was also compared to the global bacterial diversity framework of poultry [34]. Twenty-one genera, including Megamonas, Prevotella, Paraprevotella, Subdoligranulum, Hallella, Phascolarctobacterium, and minor genera representing less than ten sequences documented in the global dataset were not detected in the current pyrosequencing study. On the other hand, the current study uncovered 50 bacterial genera that were not represented in the global dataset. The major new genera include Gemmiger, Olsenella,
Moryella, Bilophila, Hydrogenoanaerobacterium, Akkermansia, Collinsella, Staphylococcus, Ruminococcus, Slackia, Sporacetigenium, and some genera each represented by less than 5 OTUs. Future studies are needed to further understand the importance and contribution of these new genera to the overall intestinal health and nutrient utilization in turkeys. It should be noted, however, that some of the identified bacterial genera contain food-borne pathogens, such as Bilophila, which is implicated in several types of infection such as perforated and gangrenous appendicitis [47]. Thus, deep sequencing analysis not only facilitates a better understanding of the turkey cecal bacteriome but also provides an opportunity to identify potential risk factors that might have been overlooked. Future studies are needed to understand the factors that govern the populations of these pathogens.

3.5. Bacterial Diversity of Turkey Ileal Mucosal Bacteriome. This study is the first reported investigation of the turkey ileal mucosal bacteriome using pyrosequencing analysis. Most of the sequencing reads turned out to be host $18 \mathrm{~S}$ rRNA gene sequences rather than bacterial 16S rRNA genes (Table 2). This is likely attributed to the low proportion of bacterial DNA in the DNA extract and also possibly the small number of PCR cycles (25 cycles). In total, only 30 bacterial 16S rRNA gene sequences were obtained from each of the two pyrosequencing runs, resulting in $<20$ OTUs (Table 2). The recovered OTUs was classified to the phyla Firmicutes, Proteobacteria, and Bacteroidetes, representing $59.3 \%, 25.0 \%$, and $6.3 \%$ of the total bacterial sequences, respectively (Figure 4). Twelve genera of bacteria were found in the ileal mucosa of the turkeys. No significant differences between the two pyrosequencing runs were observed due to the small datasets, but seven genera were identified in the first pyrosequencing run, and only three genera were identified in the second sequencing run.

Except for Lactobacillus, all the identified genera were each represented by only one OTU. Lactobacillus and Alistipes were found in both pyrosequencing runs. The coverage of bacterial diversity in the turkey ileal mucosal bacteriome was far from complete, and thus the bacterial diversity of the 
TABLE 3: Comparisons of intestinal bacterial diversity between chickens and turkeys.

\begin{tabular}{lcccc}
\hline Source & Distance level & \# of OTUs shared & $\theta_{\text {yc }}{ }^{\mathrm{b}}($ lci, hci $)$ & $0.030(0.023,0.038)$ \\
CD vs $C M$ & 0.03 & 74 & $0.052(0.034,0.068)$ & UniFrac distance \\
& 0.05 & 76 & $0.280(0.211,0.350)$ \\
& 0.20 & 31 & $0.186(0.170,0.202)$ & 0.627 \\
CD vs TD & 0.03 & 743 & $0.332(0.303,0.358)$ & 0.364 \\
& 0.05 & 758 & $0.600(0.564,0.630)$ & \\
\hline
\end{tabular}

b: $\theta_{\mathrm{yc}}=\sum_{i=1}^{S_{T}} a_{i} b_{i} /\left(\sum_{i=1}^{S_{T}}\left(a_{i}-b_{i}\right)^{2}+\sum_{i=1}^{S_{T}} a_{i} b_{i}\right)[17]$

where,

$S_{T}$ is the total number of OTUs in communities A and B;

$a_{i}$ is the relative abundance of OTU $i$ in community A;

$b_{i}$ is the relative abundance of OTU $i$ in community B.

turkey ileal mucosal bacteriome is not discussed any further in this paper. Again, future studies need to use primers that maximize amplification of bacterial 16S rRNA genes while reducing amplification of host DNA using bacteria domainspecific primers.

3.6. Comparisons between Chicken and Turkey Bacteriomes. The OTUs from the two pyrosequencing runs were combined, and the bacteriome profiles of the two species were then compared. Overall, the GI bacteriome of chickens appeared to be considerably different from that of turkeys when compared using UniFrac significance analysis $(p<$ 0.01 , data not shown). When compared on OTU richness, membership, and structure using the SONS analysis, the two GI bacteriomes were also distinct from each other, sharing only $18.6 \%$ Yue and Clayton index $\left(\theta_{\mathrm{yc}}\right.$ similarity) [17] at 0.03 distance level and $60.0 \%$ at 0.20 distance level (Table 3). As expected, greater community similarities were noted at higher phylogenetic distances.

The weighted UniFrac distances computed from the OTU representatives were used to access the structure of the poultry GI bacteriomes (Table 3). The weighted UniFrac distance was chosen over OTU-based approaches because the latter lacks the resolution to detect overlapping species between bacteriomes when datasets with low sequence coverage were used, while the former is a reliable index when comparing sequencing datasets with varied sample sizes [38]. Consistent with the analysis using SONS, the analysis using weighted UniFrac distances indicated that bacteriomes from the same GI region of the two species (chicken cecal content versus turkey cecal content) shared a greater similarity in phylogenetic structure than the bacteriomes from the different GI rejoins (ileal mucosa versus cecal content) of the same species. This is not surprising given the large differences in niche between cecal content and ileal mucosa.

Much smaller species richness was found in the ileal mucosal bacteriome of both chickens and turkeys (Table 2). About $95 \%$ of the bacteriome diversity was uncovered, but only a relatively small portion of the diversity in the ileal mucosal bacteriome of the turkeys was represented by the $16 \mathrm{~S}$ rRNA gene sequences. Because the turkey ileal mucosal bacteriome is not adequately characterized, comparison of the ileal mucosal bacteriome was not included in this paper. However, the comparison of the ileal mucosal bacterial diversity between these two bird species and a comparative phylogenetic tree are available upon request.

The distribution and relative abundance of the major bacterial genera in the cecum differed between chickens and turkeys (Figure 5). Although the cecal bacteriomes of both species shared the major phyla (Actinobacteria, Bacteroidetes, Firmicutes, and Proteobacteria), significant differences were revealed in Firmicutes, Actinobacteria, and Bacteroidetes by RDP library comparison (data not shown). Fusobacteria and Deferribacteres were minor phyla that were only identified in the chicken cecal bacteriome, whereas Verrucomicrobia and Elusimicrobia were minor phyla only identified in the turkey cecal bacteriome. At the genus level, Barnesiella and Odoribacter were significantly more abundant in the chicken cecal bacteriome while the opposite was observed for Olsenella and Rikenella in the turkey cecal bacteriome. In total, 22 major genera (each represented by $>5$ OTUs) were only identified in chicken cecal bacteriome, including Mucispirillum and Phascolarctobacterium as the most predominant genera (in descending order), whereas 23 major genera were only identified in the turkey cecal bacteriome, including Olsenella, Akkermansia, and Sporacetigenium as the most predominant genera. It is also of interest to note the different occurrence of Actinobacteria genera between the two bird species: Micrococcineae and Coriobacteriaceae were the predominant taxa in the turkey cecal bacteriome, while Bifidobacteriaceae of Actinobacteria was predominant in the chicken cecal bacteriome. Such a disparity needs to be confirmed, and their implications in host nutrition and health warrant further investigation.

As discussed earlier $[1,34,48]$, differences in host (genetics, breeds, anatomical features of the gut, physiology, etc.), litter management, and diets might be attributable to the distinct GI bacteriomes in the chicken and turkey. For example, turkeys have a larger intestinal diameter, more viscous digesta, and a slower digesta passage rate (i.e., longer retention time) than chickens [49]. These factors may lead to lower partial $\mathrm{O}_{2}$ pressure and redox potential in the gut of turkeys compared to that of chickens. Diet, however, is one the major factors affecting GI bacteriome in poultry [1]. The chickens in US commercial farms are typically fed cornsoybean-based diets that meet the NRC requirement, so were the chickens used in the present study. Thus, we compared the results from the present study with those of published studies 
where chickens were also fed corn-soybean-based diet. In the study of Lumpkin et al. [50], the bacteriome of three genetic lines of chickens were examined using DGGE and TRFLP, and Lactobacillaceae, Clostridiaceae, Enterococcaceae, and Bacteroidaceae were the major bacterial families detected in the cecum of chickens at both 4 and 35 days of age. Staphylococcaceae was also detected in multipurpose broilers. These five families were well represented in the chicken cecal bacteriome of the present study (Figure 1). Owing to the limited resolution, only five major bacterial families were detected in the study by Lumpkins et al. [50]. Our pyrosequencing analysis enabled detailed characterization of the bacteriome at OTU level. In one study on broiler chicken was challenged with Clostridium perfringens; the bacteriome was profiled using low depth coverage (6-7K sequences per sample) pyrosequencing of the V3-V5 region [51]. In the unchallenged control group fed corn-soybeanbased diet, only 2-3 major ( $>2 \%$ ) bacterial families or orders were found. At day 13, the control group was predominated by Clostridiaceae (2.41\%), Lactobacillaceae $(80.00 \%)$, and Enterobacteriaceae (11.99\%), or by Clostridiales (85.17\%) and Lactobacillaceae (7.13\%). We identify all the above major bacterial families, suggesting that the bacteriome revealed in the present study is representative of the chickens reared in commercial settings.

The diets used in studies on turkey GI bacteriome were not as well documented as those used in studies on the chicken GI bacteriome. We did a literature search of the PubMed database with keyword "turkey," "corn-soybeanbased," and "bacteriome" (or "microbiome" and "microbiota") but found no publication. However, a few studies examined the turkey GI microbiota [52, 53]. Based on Illumina sequencing of the $\mathrm{V} 3$ region of $16 \mathrm{~S}$ rRNA gene [52], the turkey cecal microbiome was dominated by Clostridia ( $90 \%$ of total bacterial sequences), while as poults grow, Bacilli, Actinobacteria, and Bacteroidia grew in predominance (5$20 \%$ ). The turkeys used in the present study were fed a cornsoybean-based diet and reared under commercial turkey management conditions. The above taxa, along with other taxa, were also detected in the present study, suggesting that the turkey GI bacteriome revealed in the present study approximates the actual GI bacteriome of turkeys reared in commercial settings. Therefore, this metagenomic study added new information to the poultry intestinal bacteriome and may facilitate future studies. It remains to be determined to what extent the distinct GI bacteriome of these two bird species contributes differently to host health and nutrient utilization.

\section{Conclusion}

A large number of genera and OTUs were found in the cecum and ileal mucosa of broiler chickens and turkeys, expanding our knowledge on the GI bacteriome of these two bird species, especially when they are reared under the dietary and managerial conditions common in North America. Some of the bacterial groups unique to each bird species might be important to host health and performance. A comprehensive knowledge of the GI bacteriome of chickens and turkeys and the differences between these bird species can be useful in modulating GI bacteriome to improve host health and growth performance.

\section{Competing Interests}

The authors declare no competing interests.

\section{Acknowledgments}

This material is based upon work that is partially supported by the National Institute of Food and Agriculture, US Department of Agriculture, under Award no. 2008-35204-18845 and by a Midwest Poultry Consortium grant.

\section{References}

[1] D. Pan and Z. Yu, "Intestinal microbiome of poultry and its interaction with host and diet," Gut Microbes, vol. 5, no. 1, pp. 108-119, 2014.

[2] M. Yegani and D. R. Korver, "Factors affecting intestinal health in poultry," Poultry Science, vol. 87, no. 10, pp. 2052-2063, 2008.

[3] S. H. Jeurissen, F. Lewis, J. D. van der Klis, Z. Mroz, J. M. J. Rebel, and A. A. H. M. ter Huurne, "Parameters and techniques to determine intestinal health of poultry as constituted by immunity, integrity, and functionality," Current Issues in Intestinal Microbiology, vol. 3, no. 1, pp. 1-14, 2002.

[4] H. U. Rehman, W. Vahjen, W. A. Awad, and J. Zentek, "Indigenous bacteria and bacterial metabolic products in the gastrointestinal tract of broiler chickens," Archives of Animal Nutrition, vol. 61, no. 5, pp. 319-335, 2007.

[5] R. D. Wagner, "Efficacy and food safety considerations of poultry competitive exclusion products," Molecular Nutrition and Food Research, vol. 50, no. 11, pp. 1061-1071, 2006.

[6] J. F. Petrosino, S. Highlander, R. A. Luna, R. A. Gibbs, and J. Versalovic, "Metagenomic pyrosequencing and microbial identification," Clinical Chemistry, vol. 55, no. 5, pp. 856-866, 2009.

[7] L. Krause, N. N. Diaz, A. Goesmann et al., "Phylogenetic classification of short environmental DNA fragments," Nucleic Acids Research, vol. 36, no. 7, pp. 2230-2239, 2008.

[8] R. A. Edwards, B. Rodriguez-Brito, L. Wegley et al., "Using pyrosequencing to shed light on deep mine microbial ecology," $B M C$ Genomics, vol. 7, article 57, 2006.

[9] P. J. Turnbaugh, R. E. Ley, M. A. Mahowald, V. Magrini, E. R. Mardis, and J. I. Gordon, "An obesity-associated gut microbiome with increased capacity for energy harvest," Nature, vol. 444, no. 7122, pp. 1027-1031, 2006.

[10] D. L. Kirchman, M. T. Cottrell, and C. Lovejoy, "The structure of bacterial communities in the western Arctic Ocean as revealed by pyrosequencing of $16 \mathrm{~S}$ rRNA genes," Environmental Microbiology, vol. 12, no. 5, pp. 1132-1143, 2010.

[11] H. Zhang, J. K. DiBaise, A. Zuccolo et al., "Human gut microbiota in obesity and after gastric bypass," Proceedings of the National Academy of Sciences of the United States of America, vol. 106, no. 7, pp. 2365-2370, 2009.

[12] N. Larsen, F. K. Vogensen, F. W. J. Van Den Berg et al., "Gut microbiota in human adults with type 2 diabetes differs from non-diabetic adults," PLOS ONE, vol. 5, no. 2, Article ID e9085, 2010. 
[13] D. Stanley, M. S. Geier, R. J. Hughes, S. E. Denman, and R. J. Moore, "Highly variable microbiota development in the chicken gastrointestinal tract," PLoS ONE, vol. 8, no. 12, Article ID e84290, 2013.

[14] D. Stanley, M. S. Geier, H. Chen, R. J. Hughes, and R. J. Moore, "Comparison of fecal and cecal microbiotas reveals qualitative similarities but quantitative differences," BMC Microbiology, vol. 15, no. 1, article no. 51, 2015.

[15] A. Qu, J. M. Brulc, M. K. Wilson et al., "Comparative metagenomics reveals host specific metavirulomes and horizontal gene transfer elements in the chicken cecum microbiome," PLoS ONE, vol. 3, no. 8, Article ID e2945, 2008.

[16] T. R. Callaway, S. E. Dowd, R. D. Wolcott et al., "Evaluation of the bacterial diversity in cecal contents of laying hens fed various molting diets by using bacterial tag-encoded FLX amplicon pyrosequencing," Poultry Science, vol. 88, no. 2, pp. 298-302, 2009.

[17] J. C. Yue and M. K. Clayton, "A similarity measure based on species proportions," Communications in Statistics. Theory and Methods, vol. 34, no. 11, pp. 2123-2131, 2005.

[18] C. Quince, A. Lanzen, R. J. Davenport, and P. J. Turnbaugh, "Removing Noise From Pyrosequenced Amplicons," BMC Bioinformatics, vol. 12, article no. 38, 2011.

[19] P. D. Schloss, D. Gevers, and S. L. Westcott, "Reducing the effects of PCR amplification and sequencing artifacts on 16s rRNAbased studies," PLoS ONE, vol. 6, no. 12, Article ID e27310, 2011.

[20] S. M. Huse, J. A. Huber, H. G. Morrison, M. L. Sogin, and D. M. Welch, "Accuracy and quality of massively parallel DNA pyrosequencing," Genome Biology, vol. 8, no. 7, article R143, 2007.

[21] V. Kunin, A. Engelbrektson, H. Ochman, and P. Hugenholtz, "Wrinkles in the rare biosphere: pyrosequencing errors can lead to artificial inflation of diversity estimates," Environmental Microbiology, vol. 12, no. 1, pp. 118-123, 2010.

[22] B. J. Haas, D. Gevers, A. M. Earl et al., "Chimeric 16S rRNA sequence formation and detection in Sanger and 454pyrosequenced PCR amplicons," Genome Research, vol. 21, no. 3, pp. 494-504, 2011.

[23] P. D. Schloss, "The effects of alignment quality, distance calculation method, sequence filtering, and region on the analysis of 16S rRNA gene-based studies," PLoS Computational Biology, vol. 6, no. 7, Article ID e1000844, p. 19, 2010.

[24] M. Kim and Z. Yu, "Variations in 16S rRNA-based microbiome profiling between pyrosequencing runs and between pyrosequencing facilities," Journal of Microbiology, vol. 52, no. 5, pp. 355-365, 2014.

[25] NRC, Nutrient Requirements of Poultry, National Academy Press, Washington, DC, USA, 8th edition, 1994.

[26] M. D. Cressman, Z. Yu, M. C. Nelson, S. J. Moeller, M. S. Lilburn, and $\mathrm{H}$. N. Zerby, "Interrelations between the microbiotas in the litter and in the intestines of commercial broiler chickens," Applied and Environmental Microbiology, vol. 76, no. 19, pp. 6572-6582, 2010.

[27] Z. Yu and M. Morrison, "Improved extraction of PCR-quality community DNA from digesta and fecal samples," BioTechniques, vol. 36, no. 5, pp. 808-812, 2004.

[28] P. D. Schloss, S. L. Westcott, T. Ryabin et al., "Introducing mothur: open-source, platform-independent, communitysupported software for describing and comparing microbial communities," Applied and Environmental Microbiology, vol. 75, no. 23, pp. 7537-7541, 2009.
[29] P. D. Schloss, "A high-throughput DNA sequence aligner for microbial ecology studies," PLoS ONE, vol. 4, no. 12, article no. e8230, 2009.

[30] W. Ludwig, O. Strunk, R. Westram et al., "ARB: a software environment for sequence data," Nucleic Acids Research, vol. 32, no. 4, pp. 1363-1371, 2004.

[31] S. M. Huse, D. M. Welch, H. G. Morrison, and M. L. Sogin, "Ironing out the wrinkles in the rare biosphere through improved OTU clustering," Environmental Microbiology, vol. 12, no. 7, pp. 1889-1898, 2010.

[32] R. C. Edgar, B. J. Haas, J. C. Clemente, C. Quince, and R. Knight, "UCHIME improves sensitivity and speed of chimera detection," Bioinformatics, vol. 27, no. 16, pp. 2194-2200, 2011.

[33] R. Larue, Z. Yu, V. A. Parisi, A. R. Egan, and M. Morrison, "Novel microbial diversity adherent to plant biomass in the herbivore gastrointestinal tract, as revealed by ribosomal intergenic spacer analysis and rrs gene sequencing," Environmental Microbiology, vol. 7, no. 4, pp. 530-543, 2005.

[34] S. Wei, M. Morrison, and Z. Yu, "Bacterial census of poultry intestinal microbiome," Poultry Science, vol. 92, no. 3, pp. 671683, 2013.

[35] Q. Wang, G. M. Garrity, J. M. Tiedje, and J. R. Cole, "Naïve Bayesian classifier for rapid assignment of rRNA sequences into the new bacterial taxonomy," Applied and Environmental Microbiology, vol. 73, no. 16, pp. 5261-5267, 2007.

[36] D. H. Huson, S. Mitra, H.-J. Ruscheweyh, N. Weber, and S. C. Schuster, "Integrative analysis of environmental sequences using MEGAN4," Genome Research, vol. 21, no. 9, pp. 1552-1560, 2011.

[37] C. Lozupone and R. Knight, "UniFrac: a new phylogenetic method for comparing microbial communities," Applied and Environmental Microbiology, vol. 71, no. 12, pp. 8228-8235, 2005.

[38] L. N. Lemos, R. R. Fulthorpe, and L. F. W. Roesch, "Low sequencing efforts bias analyses of shared taxa in microbial communities," Folia Microbiologica, vol. 57, no. 5, pp. 409-413, 2012.

[39] P. D. Schloss and J. Handelsman, "Introducing SONS, a tool for operational taxonomic unit-based comparisons of microbial community memberships and structures," Applied and Environmental Microbiology, vol. 72, no. 10, pp. 6773-6779, 2006.

[40] J. R. Cole, Q. Wang, E. Cardenas et al., "The Ribosomal Database Project: improved alignments and new tools for rRNA analysis," Nucleic Acids Research, vol. 37, supplement 1, pp. D141-D145, 2009.

[41] M. Schirmer, U. Z. Ijaz, R. D’Amore, N. Hall, W. T. Sloan, and C. Quince, "Insight into biases and sequencing errors for amplicon sequencing with the Illumina MiSeq platform," Nucleic Acids Research, vol. 43, no. 6, article no. e37, 2015.

[42] E. P. Nawrocki, D. L. Kolbe, and S. R. Eddy, "Infernal 1.0: inference of RNA alignments," Bioinformatics, vol. 25, no. 10, pp. 1335-1337, 2009.

[43] R. C. Edgar, "Search and clustering orders of magnitude faster than BLAST," Bioinformatics, vol. 26, no. 19, pp. 2460-2461, 2010.

[44] J. G. Caporaso, J. Kuczynski, J. Stombaugh et al., "QIIME allows analysis of high-throughput community sequencing data," Nature Methods, vol. 7, no. 5, pp. 335-336, 2010.

[45] W. G. Weisburg, S. M. Barns, D. A. Pelletier, and D. J. Lane, "16S ribosomal DNA amplification for phylogenetic study," Journal of Bacteriology, vol. 173, no. 2, pp. 697-703, 1991. 
[46] D. Stanley, S. E. Denman, R. J. Hughes et al., "Intestinal microbiota associated with differential feed conversion efficiency in chickens," Applied Microbiology and Biotechnology, vol. 96, no. 5, pp. 1361-1369, 2012.

[47] E. J. Baron, "Bilophila wadsworthia: a unique gram-negative anaerobic rod," Anaerobe, vol. 3, no. 2-3, pp. 83-86, 1997.

[48] L. Wang, M. Lilburn, and Z. Yu, "Intestinal microbiota of broiler chickens as affected by litter management regimens," Frontiers in Microbiology, vol. 7, article 593, 2016.

[49] S. Palander, M. Näsi, and P. Palander, "Digestibility and energy value of cereal-based diets in relation to digesta viscosity and retention time in turkeys and chickens at different ages estimated with different markers," Archives of Animal Nutrition, vol. 64, no. 3, pp. 238-253, 2010.

[50] B. S. Lumpkins, A. B. Batal, and M. D. Lee, "Evaluation of the bacterial community and intestinal development of different genetic lines of chickens," Poultry Science, vol. 89, no. 8, pp. 1614$1621,2010$.

[51] Y. O. Fasina, M. M. Newman, J. M. Stough, and M. R. Liles, "Effect of Clostridium perfringens infection and antibiotic administration on microbiota in the small intestine of broiler chickens," Poultry Science, vol. 95, no. 2, pp. 247-260, 2016.

[52] J. L. Danzeisen, J. B. Clayton, H. Huang et al., "Temporal relationships exist between cecum, ileum, and litter bacterial microbiomes in a commercial turkey flock, and subtherapeutic penicillin treatment impacts ileum bacterial community establishment," Frontiers in Veterinary Science, vol. 2, 2015.

[53] J. L. Danzeisen, A. J. Calvert, S. L. Noll et al., "Succession of the turkey gastrointestinal bacterial microbiome related to weight gain," PeerJ, vol. 2013, no. 1, article no. e237, 2013. 

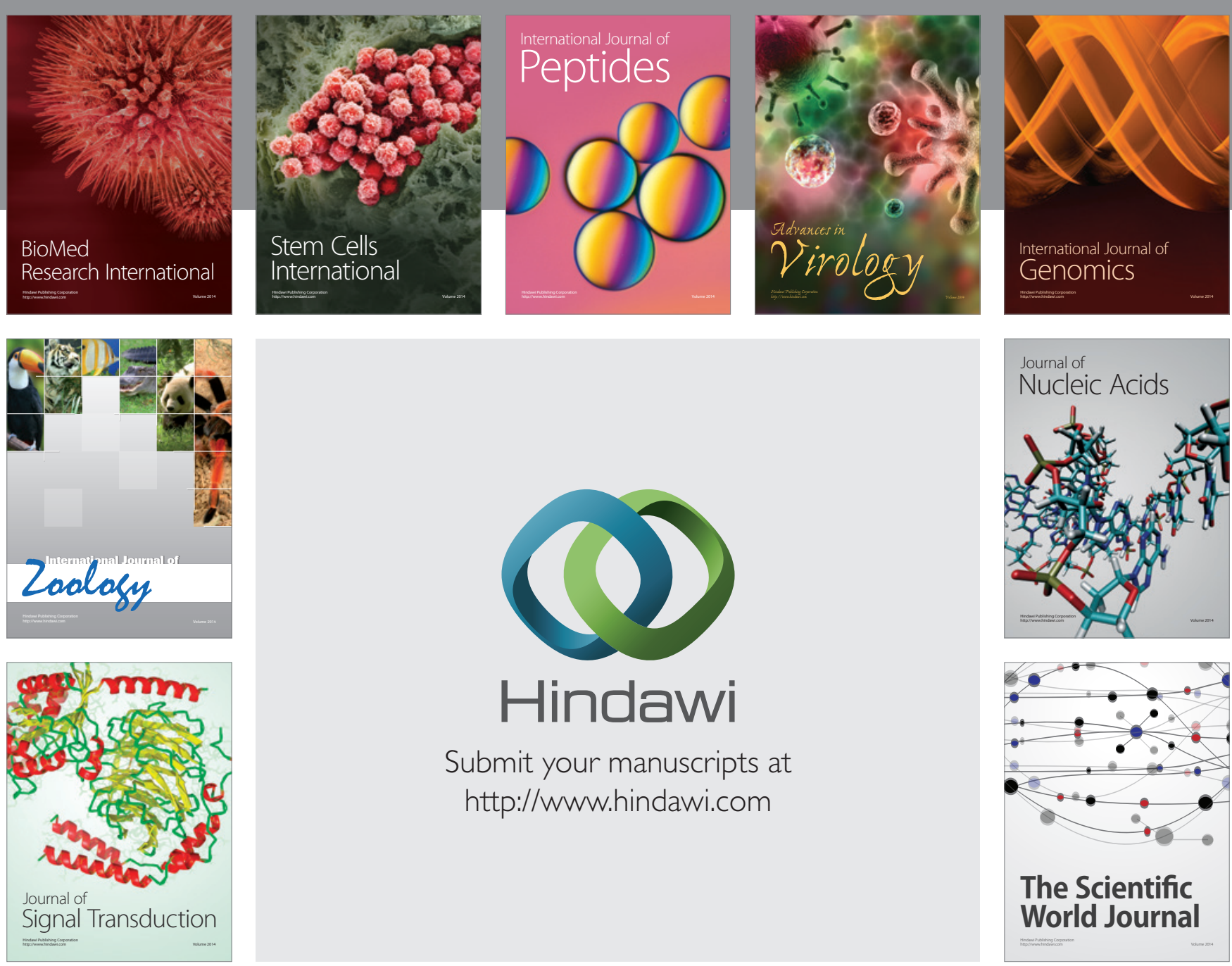

Submit your manuscripts at

http://www.hindawi.com
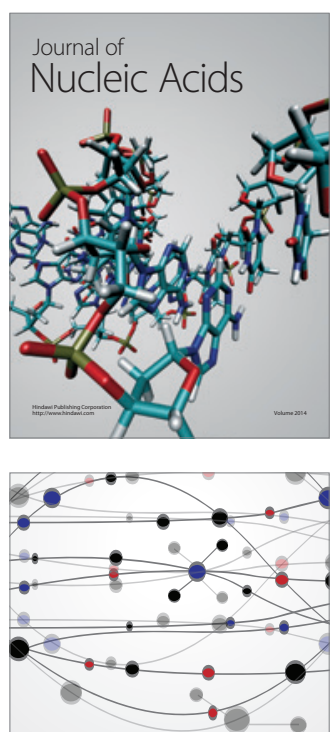

The Scientific World Journal
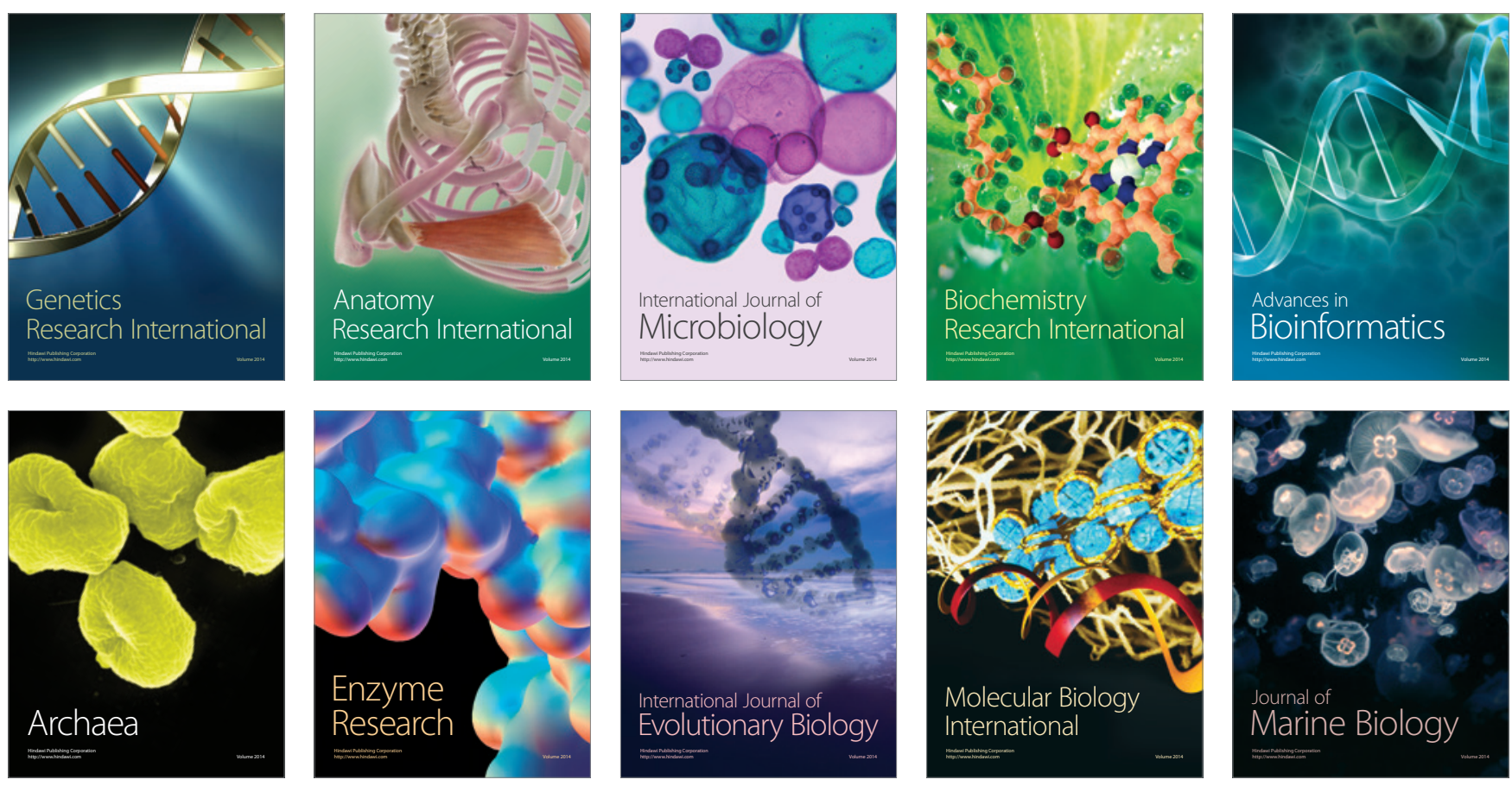